\title{
Breads formulated with evolutionary populations of wheat heritage varieties on postprandial glycaemic and insulin responses in healthy subjects
}

\author{
$\underline{\text { Margherita Dall'Asta }}{ }^{1}$, Rossella Dodi $^{2}$, Giuseppe Di Pede ${ }^{2}$, Mia Marchini $^{3,1}$, Silvia Folloni ${ }^{3}$ and \\ Francesca Scazzina ${ }^{1}$ \\ ${ }^{1}$ Department of Food and Drug, University of Parma, PARMA, Italy, \\ ${ }^{2}$ Department of Veterinary Science, University of Parma, PARMA, Italy and \\ ${ }^{3}$ Open Fields srl, PARMA, Italy
}

\begin{abstract}
Triticum heritage varieties and ancient species are gaining interest of consumers due to the perception of a healthier nutrition profile than other modern wheat. Although still limited, several findings highlighted the real potential beneficial role of ancient wheat-based products consumption on several cardiometabolic factors ${ }^{(1)}$. However, a lack of information is still present, especially for their effect on postprandial glycaemia. Evolutionary populations (EPs) are plants within the same species in one field characterized by a high level of genetic diversity ${ }^{(2)}$. EPs are evolving as a function of several environmental variables and in turn characterized by a high level of adaptability and biodiversity. EPs can drive agronomic advantages, especially under organic and low-input agricultural conditions, or in less favourable growing conditions ${ }^{(3)}$. The aim of the present study, which is a part of a more comprehensive project called "BIO ${ }^{2,(4)}$, was to evaluate the impact of breads made with EPs of heritage varieties and a modern variety, all produced under organic farming in mountain areas of Emilia Romagna Region (Italy), on postprandial glycaemic and insulin responses. The study is a randomized controlled trial in which 13 healthy subjects were enrolled for consuming 8 non-commercial breads made either with EPs (EPs named "BIO" " and "GROSSI" - of local heritage varieties, and EP "Solibam") or with a modern variety (Bologna) produced with a standard recipe, but 2 different leavening techniques ( $S$. Cerevisiae and sourdough). Subjects consumed breads in a portion containing $50 \mathrm{~g}$ of available carbohydrates and blood was collected for quantifying glycaemia and insulin during 2 hours. Incremental area under curves (IAUCs) and maximum peaks were compared among treatments. No differences were found in IAUCs and peaks for both the markers, showing that none of the tested EPs was effective in lowering glycaemic and insulin responses compared to the modern variety. Moreover, sourdough did not determine a lower blood glucose and insulin within the same type of flour, which is in contrast with previous studies describing the role of sourdough in lowering glycaemic response. Future studies will better elucidate the possible implication of consuming ancient wheat-based products on the modulation of post-prandial glucose metabolism.
\end{abstract}

This study has been funded by Measure 16.1.01 of the Rural Development Programme 2014-2020 of the Emilia-Romagna Region (Italy).

\section{Conflict of Interest}

There is no conflict of interest

\section{References}

1. Dinu et al. (2018) J Nutr Biochem 52,1-9.

2. FAO (2009). International treaty on plant genetic resources for food and agriculture

3. Raggi et al. (2017) Field Crops Res 204, 76-88.

4. www.bioalquadrato.it 